Vol. 72, N. ${ }^{\circ}$ II5 (mayo 2020), 33-49

\title{
ENDOGENEIDAD MONETARIA EN UNA ECONOMÍA DOLARIZADA: CASO ECUADOR
}

MONETARY ENDOGENEITY IN A DOLLARIZED ECONOMY: ECUADOR CASE

\author{
ANTHONY POZO BARRERA, DOMÉNICA CÁRDENAS LÓPEZ \\ Investigadores independientes
}

Recepción manuscrito: 13 de abril 2020

Aceptación versión final: 18 de mayo de 2020

\begin{abstract}
RESUMEN La presente investigación detalla las principales características de la endogeneidad monetaria del Ecuador, basándose en las vertientes horizontalista, estructuralista y de preferencia de liquidez de la corriente poskeynesiana. Utilizando un modelo de Vectores Autorregresivos (VAR) y pruebas de causalidad de Granger, se determina que para el periodo 2007-2019 la endogeneidad de la oferta monetaria satisface los postulados de la vertiente horizontalista (acomodatista), puesto que se pudo constatar una causalidad unidireccional del crédito bancario a la base monetaria, una causalidad indirecta del crédito bancario a la oferta monetaria y una causalidad unidireccional del PIB real a la oferta monetaria. ${ }^{1}$

PALABRAS CLAVE Endogeneidad monetaria, teoría poskeynesiana, modelo VAR, dolarización, crédito bancario.
\end{abstract}

ABSTRACT This research details the main characteristics of the monetary endogeneity of Ecuador, based on the horizontalist, structuralist and liquidity preference aspects of the post-Keynesian current. Using a model of Autoregressive Vectors (VAR) and Granger causality tests, it is determined that for the period 2007-2019 the endogeneity of the money supply satisfies the postulates of the horizontalist (accommodative) side, since it was found a one-way causality of bank credit to the monetary base, an indirect causality of bank credit to the money supply and a oneway causality of real GDP to the money supply.

KEYWORDS Endogenous monetary, post-Keynesian theory, vAR model, dollarization, bank credit.

JEL CODES E12, $\mathrm{C}_{32}, \mathrm{E}_{42}, \mathrm{E} 51$.

\section{INTRODUCCIÓN}

Existe una amplia variedad de estudios orientados a determinar la endogeneidad o exogeneidad de la oferta monetaria, este debate tiene origen desde los pensadores clásicos y sigue vigente hasta el presente, dichos estudios se enfocan en la validación de sus hipótesis teniendo 
como base una amplia evidencia empírica y la aplicación de técnicas econométricas. El debate actual no solo se centra en la determinación de la endogeneidad o exogeneidad de la oferta monetaria, más bien, el tema de especial relevancia gira en torno a la determinación del tipo de endogeneidad que siguen los regímenes monetarios de los países.

Para el caso ecuatoriano, este debate toma cierta particularidad, ya que al ser una economía dolarizada se limita su capacidad para emitir y manejar su propia moneda, además de que los mecanismos que podrían incidir en el manejo de su oferta monetaria aún no han sido explorados a profundidad. Tomando en cuenta esta limitación, Davidson (2002) argumenta que buena parte de la liquidez de la economía ecuatoriana (y ligado a ello, su desempeño) va condicionado a su capacidad de obtener divisas del exterior, ya sea por un superávit en la cuenta corriente, préstamos provenientes del exterior o la capacidad institucional que se tenga para repatriar fondos depositados en el extranjero de ciudadanos residentes.

Adicional, la Constitución de la República del Ecuador (2008) y el Código Orgánico Monetario y Financiero (2016), mantienen la concepción de que la oferta monetaria se determina exógenamente a través de la balanza de pagos, por lo cual se constituye como uno de los principales objetivos de la política económica. Este precepto ha sido refutado por el estudio de Pérez y Guncay (2019), en donde a través de técnicas econométricas se demuestra que la oferta monetaria en el Ecuador se determina de manera endógena, es decir, que no está determinada por el resultado de la balanza de pagos, sino a través del desempeño mismo de la economía. Sin embargo, la determinación de la endogeneidad monetaria no es suficiente para entender de mejor manera el proceso de creación monetaria, es necesario también entender las características de esta endogeneidad y cuál es el apego teórico que esta tiene con las principales vertientes de la corriente poskeynesiana.

Por lo cual, el presente estudio parte de los resultados hallados en la investigación de Pérez y Guncay (2019), pero posee ciertas diferencias tanto en su metodología, variables utilizadas y en su alcance. Primero, la técnica utilizada en el presente estudio es la estimación de un modelo de Vectores Autorregresivos (VAR) en forma reducida y el estudio antes mencionado utiliza un modelo VAR recursivo, es decir, el primer modelo no tiene restricciones en su especificación mientras que el segundo sí está sujeto a ciertas restricciones.

La segunda diferencia es en las variables que se utilizaron para la determinación de la endogeneidad/exogeneidad, en el presente estudio se utilizan la base monetaria, oferta monetaria, crédito bancario, multiplicador monetario, PIB real y el índice de precios al consumidor; mientras que en el estudio antes mencionado para tal determinación se utiliza al crédito y la liquidez total.

La tercera tiene que ver con su alcance, el presente estudio además de confirmar o rechazar la endogeneidad/exogeneidad de la oferta monetaria, también testea hipótesis monetarias de diversas concepciones de endogeneidad monetaria provenientes de la corriente poskeynesiana; en cambio, el estudio antes mencionado se ocupa de la determinación de la endogeneidad/exogeneidad monetaria y la relación existente entre la liquidez total y la balanza de pagos.

El presente estudio consta de la introducción que se acaba de presentar, una revisión de literatura en donde consta la caracterización de las diversas concepciones de exogeneidad/endogeneidad monetaria, además que se detallan los estudios relacionados, la metodología aplicada y datos estadísticos utilizados, los principales resultados obtenidos con su respectivo análisis, 
una discusión de los principales resultados hallados en comparación con otros estudios relacionados y las conclusiones del estudio.

\section{REVISIÓN DE LITERATURA}

OFERTA DE DINERO: EXOGENEIDAD Y ENDOGENIDAD

La concepción de la determinación de la oferta de dinero ha sido un tema ampliamente debatido por los economistas, la teoría de exogeneidad del dinero proviene desde los pensadores clásicos con David Hume en un inicio y es defendida en la actualidad por la escuela monetarista, cuyo principal exponente fue Milton Friedman, pues éste afirma que «en Estados Unidos, la Reserva Federal controla la oferta de dinero» (Brue and Grant, 2009, págs. 500-501); mientras que la teoría de endogenidad del dinero fue postulada como una respuesta a la concepción clásica especialmente por los economistas de la corriente poskeynesiana con Nicolás Kaldor a la cabeza de esta crítica (Mántey, 1997), a continuación se detallan ambas teorías con sus particularidades.

\section{MONETARISMO}

El análisis monetarista centra su atención tanto en el corto como en el largo plazo. Para el corto plazo definen que existe un ente regulador (Banco Central) que es el encargado de determinar la cantidad de dólares que se ofertan en una economía, esta característica se la conoce como la exogeneidad de la oferta monetaria, reconocen que existe un equilibrio en el nivel de precios cuando la oferta monetaria es igual a la demanda de dinero y que esta demanda de dinero es estable basándose en la ecuación cuantitativa del dinero (Mántey, 1997).

Para el corto plazo también definen que el dinero no es neutral, pues postulan que, en situaciones de desequilibrios, un incremento de la oferta monetaria podría tener incidencia en variables reales como la inversión o el ingreso nacional, ya que esto hace que las personas tengan un dinero excedente y ello fomenta a un mayor gasto en la economía, causando un desplazamiento de la demanda agregada y llevando nuevamente a la economía dentro de la senda del equilibrio (Vega Miranda y Lechuga, 2018).

En el largo plazo, los monetaristas mantienen su concepción de exogeneidad del dinero y una demanda de dinero estable basándose en la ecuación cuantitativa del dinero, pero además postulan que el dinero sí es neutral en el largo plazo a causa del ajuste de las expectativas de los agentes (Friedman, 1977), incluyen en sus análisis el desempleo y postulan que un incremento de la oferta monetaria puede incidir en la reducción del desempleo en un primer momento, pero que en el largo plazo dado un incremento de precios causado por la expansión monetaria y salarios rígidos de los trabajadores beneficiados en el primer momento, genera que este salario resulte insuficiente para el nuevo nivel de precios y que los trabajadores presionen por salarios más altos, pero esto genera que el nivel de empleo vuelva su estado «natural» (Mántey, 1997).

\section{TEORÍA MONETARIA DE LA PRODUCCIÓN}

Entre finales de 1930 e inicios de 1932, se marca un cambio en el pensamiento de J. M. Keynes. En otoño de 1932, el título de sus conferencias se convierte en «la teoría monetaria de la 
producción», y después en «los parámetros de la economía monetaria de producción». En este periodo, Keynes se encontraba en una lógica de proyecto radical, en una fase de construcción de una nueva teoría macroeconómica y monetaria de la producción (Piégay y Monchon, 2006).

En 1933, Keynes rechaza el concepto de dinero neutral, que involucra a los intercambios. La incertidumbre generalizada y el dinero afectan, por el contrario, a las decisiones de los agentes y al funcionamiento de todo el sistema. Este análisis aparece concretamente en sus escritos Una teoría monetaria de la producción, donde estudia las diferencias entre las economías monetarias y las economías de intercambio real. La primera se refiere a aquellas economías donde el dinero juega un papel por sí mismo, afectando a las motivaciones y las decisiones, de modo que no se puede predecir el curso de los acontecimientos y el papel que juega concretamente el dinero; la segunda se refiere en aquellas economías que utilizan el dinero únicamente como parte neutral entre transacciones de bienes y de activos reales, y que no permite inmiscuirse en las motivaciones y decisiones (Keynes, 1993).

Las diferencias entre economía de intercambio real y economía monetaria, han sido a lo largo del tiempo subestimadas, llevando a la práctica muchas conclusiones y políticas equivocadas, producto de adaptaciones hipotéticas de una economía de salarios reales a la economía monetaria del mundo real, lo cual es un error significativo, puesto que realizar este tipo de adaptaciones viene considerado sumamente complejo e incluso imposible, sin la ayuda de una teoría bien elaborada de una economía monetaria (ibid.).

\section{TEORÍA POSKEYNESIANA}

Entre los teóricos heterodoxos existe un consenso sobre la endogeneidad del dinero, es decir, que no atribuyen el suministro y control cuantitativo de éste a un ente regulador exógeno (Banco Central), sino que argumentan que el dinero es creado por el proceso de financiación del gasto privado, además de rechazar la idea del crecimiento estable de la oferta monetaria propuesta por los monetaristas con Friedman a la cabeza (Wray, 2007).

Este proceso de creación del dinero sucede cuando se otorga créditos tanto a empresas u hogares según sus necesidades de producción o consumo, esto último es la característica completamente endógena de la oferta de dinero, además señalan que en la actualidad los bancos centrales son incapaces de controlar la cantidad de dinero per se, sino que utilizan a la tasa de interés a corto plazo para controlarlo basando la fijación de esta tasa con algún objetivo de inflación (Bain and Howells, 2003).

Si bien los poskeynesianos tienen un consenso sobre la endogenidad de la oferta monetaria, surgieron tres corrientes dentro de esta concepción: los horizontalistas (acomodatistas), estructuralistas y los de la preferencia de liquidez; dichas corrientes no tienen grandes diferencias entre sí, pues la parte sustantiva de sus diferencias se centra en el comportamiento de los agentes económicos y el alcance de las herramientas de política monetaria del Banco Central (Moore, 1991), sus particularidades serán expuestas a continuación.

- horizontalistas (acomodatistas). El enfoque horizontalista, además de determinar que el crédito bancario es el mecanismo creador de la moneda, analizan el rol del Banco Central, la tasa de interés y la preferencia por la liquidez en este proceso. Sobre el Banco Central, caracterizan que es el encargado de proveer completamente las necesidades de reservas y de 
jugar un papel de prestamista de última instancia a los bancos comerciales; la salud del sistema financiero hace que la banca central se «acomode» a las necesidades de reservas de estos bancos y mantenga una actitud pasiva frente a la demanda de crédito (Garzón, 2009). La tasa de interés de corto plazo es el mecanismo de regulación de la demanda de crédito bancario, esta variable es manejada de manera exógena por las autoridades monetarias y tiene por objetivo evitar los problemas de liquidez, es decir, que la oferta monetaria dada por el crédito bancario tendrá una relación completamente elástica con la tasa de interés (ibid). Por último, este enfoque no otorga un papel relevante a la preferencia por la liquidez de los agentes, pues se resalta que «La preferencia del público por mantener dinero no juega ningún papel» (Lavoie, 1992, pág. 193); esto genera que los bancos no tengan que limitar la cantidad de crédito que otorgan, pues no se enfrentan al dilema de si mantener su liquidez o su rentabilidad y más bien mantienen un comportamiento pasivo ante la demanda del crédito (Garzón, 2009).

- estructuralistas. Esta perspectiva también centra su atención en el rol del Banco Central, la tasa de interés y la preferencia por la liquidez en el proceso de oferta monetaria. Discrepa en el papel de la banca central, pues argumenta que ésta solo atenderá parcialmente la demanda de reservas por parte de la banca comercial y no en su totalidad como postulan los horizontalistas; esta acción le da a la banca central cierto control sobre la oferta de reservas, además señalan que a medida que se incremente la demanda de crédito bancario también se incrementará la tasa de interés, es decir, que la función de oferta monetaria será de pendiente ascendente, así, la demanda de crédito y la tasa de interés tendrán una relación inelástica (Palley, 1996). También niegan que la banca central tenga solamente el control de la tasa de interés a corto plazo para evitar problemas de liquidez, sino que además de la tasa de interés a corto plazo, la banca central también puede gestionar la base monetaria para enfrentar estos problemas; incluso postulan que podrían utilizar estas herramientas para algún otro objetivo (Palley, 1998); además, otorgan un reconocimiento a la banca comercial al momento de gestionar sus pasivos, pues argumentan que dichos bancos, ante las restricciones de suministro de reservas por parte de la banca central, buscarán fondos que sean baratos especialmente cuando se incrementen las tasas de interés (Palley, 1996). A diferencia de la perspectiva horizontalista, esta postura sí toma en consideración el papel de la preferencia por la liquidez, argumentan que la incertidumbre es una característica importante dentro del sistema económico y que también debe tomarse en cuenta por la banca, ya que en esta estructura éstos sí se enfrentan a la disyuntiva entre rentabilidad o liquidez (Paula, 2003), por lo cual la perspectiva de los agentes sobre el mercado si podría determinar la demanda de crédito y con ello la oferta monetaria (Garzón, 2009).

- PREFERENCIA POR LA LIQUidez. Uno de los principales autores en abordar la incertidumbre dentro del estudio de la economía fue Keynes (1936), se entiende como preferencia por la liquidez a la demanda de dinero y detalla tres motivos por los cuales la incertidumbre puede afectar a la preferencia por la liquidez de los agentes: (1) el motivo transacción, que es la preferencia por el efectivo de hogares y de empresas para realizar sus transacciones comerciales, (2) motivo precaución, que es el deseo de la seguridad con respecto a las expectativas del futuro, y (3) motivo especulación, que es el deseo de obtener ganancias por intuir 
lo que el futuro traerá consigo mejor que el mercado. Adicional, Keynes, en su Tratado del dinero, identifica un motivo adicional como determinante de la demanda de dinero: demanda financiera de dinero, entendida como el crédito otorgado por parte de los bancos ante la demanda de dinero que requieren las empresas para ejecutar sus planes de inversión (Pastrana, 2018). La incertidumbre es una de las razones que justifica la existencia de la preferencia de la liquidez de los agentes, dentro de la endogeneidad de la oferta monetaria este postulado indica que las expectativas/incertidumbre de los agentes pueden llegar a influir en la determinación de la tasa de interés de corto plazo de los bancos centrales, y estas expectativas surgen como respuesta al desempeño de la economía en su conjunto, es por este motivo que el comportamiento de los agentes podría llegar a incidir tanto en la tasa de interés a corto plazo como en la demanda del crédito bancario y con ello la oferta monetaria (Chick and Dow, 2002).

\section{TRABAJOS PREVIOS}

En las últimas décadas, se han realizado varias investigaciones para determinar la hipótesis de endogeneidad de la oferta monetaria, un rasgo distintivo en la literatura encontrada es la metodología aplicada, pues en la mayoría de los estudios se utilizan modelos var, acompañados de pruebas de causalidad de Granger.

Jafar Haghighat (2011) realizó un estudio donde el objetivo fue determinar si la oferta monetaria es endógena, ya sea por el comportamiento del Banco Central de Irán o si ésta es endógena en concordancia con los postulados poskeynesianos, utilizando datos desde 1968 hasta el 2009. El autor realizó pruebas empíricas de raíz unitaria, test de cointegración de Johansen y si las variables se mostraban cointegradas empleó modelos de corrección de errores de vectores (VEC), además del test de causalidad de Granger, para comprobar si existe una causalidad unidireccional o bidireccional. Todo ello se realizó mediante el uso de las variables de crédito por parte de los bancos, la base monetaria, la oferta monetaria y el ingreso. El autor finalizó la investigación determinando que la oferta monetaria en Irán es endógena.

De igual manera, Francisco Alvarado (2011) elaboró un estudio donde se evaluó la hipótesis poskeynesiana del dinero endógeno para Colombia en el periodo 1982-2009. En este trabajo, toma como variables los agregados monetarios м2 у м 3, además de la cartera neta del sistema financiero. Para determinar si la causalidad entre las variables propuestas surge de los planteamientos poskeynesianos, se evalúan mediante la comprobación de relaciones de causalidad estadística, específicamente, se realizó la estimación de modelos var. El autor concluye que los resultados obtenidos soportan el planteamiento horizontalista y, por ende, el enfoque poskynesiano, ratificando la existencia de una determinación endógena de los agregados monetarios.

Así mismo, Shanmugam et al. (2003), desarrollaron un estudio donde se analiza la hipótesis de la oferta de dinero endógeno en el entorno de Malasia desde 1985 hasta el 2000. En este trabajo, se toma como variables el ingreso y el agregado monetario M3. Los resultados respaldan la hipótesis poskeynesiana, según la cual la oferta de dinero en Malasia fue endógena durante el periodo de estudio, también demostraron una relación de cointegración a largo plazo. Se concluye, además, que los resultados obtenidos no parecían apoyar el enfoque estructuralista ni tampoco mostraban a oposición al mismo. 
Rodríguez et al. (2000), realizaron un trabajo donde se analiza la relación de causalidad entre diferentes agregados monetarios en España durante el periodo 1978 hasta 1998 con el propósito de determinar si la oferta monetaria es exógena o endógena. Para ello, se tomaron como variables de estudio a los activos de caja del sistema bancario o la base monetaria, al M3 y a la oferta monetaria. Dentro de este estudio se emplearon dos técnicas econométricas: la prueba de causalidad de Granger y el modelo vec. Los resultados obtenidos dentro de la investigación indican que, para ese periodo de estudio en España, la oferta de dinero es de carácter endógeno.

De manera similar, Meutia Safrina Rachma (2010), realizó un estudio donde se evaluó si la oferta monetaria en Indonesia era una variable endógena o una variable exógena. Dentro de esta investigación, se realizó la estimación de modelos var usando datos mensuales desde 1997 hasta el 2010. Este estudio utilizó las variables M1, M2, la base monetaria (MO) y el índice de precios al consumidor (IPC). El resultado de la estimación muestra que la oferta monetaria en Indonesia en los periodos seleccionados es una variable endógena. El Banco Central no tiene poder de control sobre la oferta monetaria, éste solo puede mantener la estabilidad y controlar el movimiento de M2.

Una forma resumida de expresar los diversos enfoques de las teorías monetarias previamente detalladas y las posibles causalidades entre las variables encontradas en la literatura estudiada se detallan en la tabla explicativa (ver Tabla 1).

\section{DOLARIZACIÓN Y ENDOGENEIDAD MONETARIA}

La primera moneda acuñada en el país por un ente estatal fue el sucre, que vio la luz cuando se creó el Banco Central del Ecuador (ВСЕ) en 1927, a raíz de esto el país pudo empezar a aplicar política monetaria desde el Estado. En 1980, el sucre se había depreciado frente al dólar estadounidense (USD) y llegó a cambiarse a 28 sucres por 1 USD; para el año 2000, la moneda nacional se llegó a cambiar a 25.000 sucres por 1 USD, además de que el país vivía la inflación más alta registrada en toda la historia (Villalba, 2019).

Los motivos que explican el mal desempeño monetario hasta el año 2000 son diversos, existieron choques exógenos como el incremento de las tasas internacionales, crisis de deuda externa, el Fenómeno del Niño y terremotos, el desplome de los precios del petróleo y de algunos de los principales productos de exportación, además de la guerra no declarada con el Perú (ibid.).

Adicional, existieron algunos factores de influyeron directamente en la dinámica monetaria, el primero fue la «sucretización» de los años 80, que consistió en un subsidio para empresarios locales que se endeudaron en el extranjero en dólares, para lo cual hubo una importante emisión monetaria que se tradujo en inflación y el «salvataje bancario» de fines de los años 90, que destinó cerca del 26\% del PIB de diciembre 1998 a diciembre 1999 para tratar de salvar las principales entidades financieras del colapso ( $i b i d$.$) .$

Es en medio de este panorama que para el año 2000 se dio paso la dolarización oficial de la economía ecuatoriana, en la cual el BCE perdía la capacidad de emisión monetaria, esta medida tenía como objetivos frenar la alta volatilidad cambiaria, controlar la creciente inflación, reactivar el aparato productivo y enviar señales de seguridad y cumplimiento de obligaciones a la comunidad internacional (Naranjo, 2003). 
Sin embargo, la dolarización para el Ecuador demostró muchas limitantes con el paso del tiempo, en primera instancia la pérdida de su política cambiaria y monetaria complica la posibilidad de aplicar políticas contracíclicas si se necesitan; segundo, el país está mucho más vinculado a un ciclo económico diferente al propio, lo cual hace que sea muy vulnerable a decisiones que se puedan tomar en la Reserva Federal de EE. UU. (FED) y que afecten al Ecuador, sin posibilidad de reclamo; tercero, sufre de constantes déficits comerciales por pérdida de competitividad frente a sus vecinos y cuarto, una crisis en los EE. UU. puede tener efectos mayores en una economía dolarizada que si no lo estuviera (Acosta, 2001).

Bajo este contexto, Guncay y Pérez (2019) realizaron una investigación para evaluar la hipótesis poskeynesiana de endogeneidad de dinero en Ecuador, mediante la estimación de modelos var y pruebas de causalidad de Granger, utilizando como variables a la liquidez total (M2) y al crédito bancario, donde se pudo determinar que la oferta de dinero en el Ecuador es endógena. Dichos resultados se obtienen debido a que posee un efecto unidireccional positivo desde el crédito hacia la liquidez total y del crédito hacia los depósitos, esto indica que las alteraciones en el nivel de crédito no están totalmente condicionados a la oferta monetaria, más bien, éstas se encuentran fuertemente relacionadas a la actividad económica, así como la corriente poskeynesiana señala $(i b i d)$.

Por otro lado, dentro de esta investigación se evidenció que la balanza de pagos no es el principal determinante de la liquidez en la economía ecuatoriana, se llegó a esta conclusión mediante la ejecución de la función de impulso respuesta, donde se obtiene como resultado que un shock acumulado normalizado a una desviación estándar sobre la balanza de pagos solo incrementa alrededor del $1 \%$ la oferta monetaria por un periodo de seis trimestres (ibid). En contraste, utilizando la misma técnica antes señalada, se obtuvo que un shock acumulado normalizado a una desviación estándar sobre el crédito bancario incrementa la liquidez de la economía en aproximadamente $2,3 \%$ por un periodo de cinco trimestres, es decir, el crédito bancario posee un mayor efecto sobre la oferta monetaria con respecto a los resultados de la balanza de pagos (ibid).

\section{METODOLOGÍA Y MODELO}

En la presente sección se detalla la estrategia metodológica utilizada para la determinación de las diversas hipótesis monetarias exógenas/endógenas, éstas provienen de la vertiente monetarista, horizontalista (acomodatista), estructuralista y de preferencia de la liquidez antes detalladas. También se detallan las fuentes de información utilizadas, variables seleccionadas y la especificación del modelo utilizado.

\section{MODELO DE VECTORES AUTORREGRESIVOS (VAR)}

El modelo var es un modelo de ecuaciones simultáneas que está conformado por un sistema de ecuaciones en forma reducida y sin restringir; la característica de este modelo es que toma a todas sus variables como endógenas, es decir, que las variables dependen unas de otras (Novales, 2017). Se seleccionó el modelo var para el estudio porque este permite identificar una dinámica causal entre todas las variables, es decir, este modelo permite aplicar test 
Tabla 1. Síntesis de la causalidad de los diferentes enfoques de las teorías monetarias

\begin{tabular}{lllll}
\hline Monetarismo & Horizontalista (Acomodatista) & Estructuralista & Preferencia por la liquidez \\
\hline $\mathrm{BM} \Rightarrow \mathrm{CB}$ & $\mathrm{CB} \Rightarrow \mathrm{BM}$ & $\mathrm{CB} \Leftrightarrow \mathrm{BM}$ & $\mathrm{CB} \Leftrightarrow \mathrm{M} 1$ \\
\hline $\mathrm{M} 1 \Rightarrow \mathrm{CB}$ & $\mathrm{CB} \Rightarrow \mathrm{M} 1$ & $\mathrm{CB} \Leftrightarrow \mathrm{MM}$ & \\
$\mathrm{M} 1 \Rightarrow \mathrm{Y}$ & $\mathrm{Y} \Leftrightarrow \mathrm{M} 1$ & $\mathrm{Y} \Leftrightarrow \mathrm{M} 1$ & \\
\hline
\end{tabular}

Nota: base monetaria (вм), oferta monetaria (М1), crédito bancario (Св), PIB real (Y), multiplicador monetario (Mм). Fuente: Haghighat (2011, pág. 66).

de causalidad de Granger, para esto es necesario que todas las variables seleccionadas sean estacionarias, dejando como resultado que $X_{t}$ debe causar en el sentido de Granger a $Y_{t}$ o viceversa (Gujarati and Porter, 2010).

El modelo var en su forma reducida y con rezagos se puede expresar de la siguiente manera:

$$
Y_{t}=m+A_{1} Y_{t-1}+\ldots+A_{p} Y_{t-p}+u_{t}
$$

Y su representación matricial sería:

$$
Y_{t}=m+A Y_{t-1}+u_{t}
$$

En donde:

$$
\begin{aligned}
& Y_{t}=\text { vector de variables consideradas en el modelo } \\
& m=\text { vector de constantes de orden } k x_{1} \\
& Y_{t-1}=\text { vector de variables rezagadas en un periodo } \\
& A=\text { matriz de parámetros } \\
& u_{t}=\text { vector del término error }
\end{aligned}
$$

\section{VARIABLES Y DATOS}

En base a la literatura consultada las variables utilizadas para la estimación del modelo son: base monetaria (BM), oferta monetaria (M1), multiplicador monetario ( $\mathrm{MM}$ ), total créditos al sector privado (Св), PIB real (y) y el índice de precios al consumidor (IPC), todas las variables utilizadas tienen año base 2007 y se clasificó la información trimestralmente, las series van desde el primer trimestre del año 2007 al cuarto trimestre del 2019, con excepción de las variables Y e IPC, cuya serie llega hasta el tercer trimestre del 2019.

Las variables BM, M1, MM, Св, fueron construidas a partir de la Información Estadística Mensual del вСE, específicamente del apartado Principales Indicadores Monetarios y Financieros se obtuvo valores trimestrales de las series y éstas contienen valores nominales. La variable y fue obtenida a través del apartado del Sector real: Producto Interno Bruto; una vez obtenida la serie trimestral en valores nominales se lo dividió para el deflactor del PIB, obteniendo así la serie en términos reales. Por último, la serie IPC es un índice obtenido trimestralmente, provista por el Instituto Nacional de Estadísticas y Censos (INEC). 
Para el presente estudio, una vez que se describieron las variables utilizadas, se presenta el modelo utilizado en su forma matricial:

$$
\left(\begin{array}{c}
B M_{t} \\
C B_{t} \\
M M_{t} \\
Y_{t} \\
I P C_{t} \\
M 1_{t}
\end{array}\right)=\left(\begin{array}{l}
m_{1} \\
m_{2} \\
m_{3} \\
m_{4} \\
m_{5} \\
m_{6}
\end{array}\right)+\left(\begin{array}{llllll}
a_{11} & a_{12} & a_{13} & a_{14} & a_{15} & a_{16} \\
a_{21} & a_{22} & a_{23} & a_{24} & a_{25} & a_{26} \\
a_{31} & a_{32} & a_{33} & a_{34} & a_{35} & a_{36} \\
a_{41} & a_{42} & a_{43} & a_{44} & a_{45} & a_{46} \\
a_{51} & a_{52} & a_{53} & a_{54} & a_{55} & a_{56} \\
a_{61} & a_{62} & a_{63} & a_{64} & a_{65} & a_{66}
\end{array}\right)\left(\begin{array}{c}
B M_{t-1} \\
C B_{t-1} \\
M M_{t-1} \\
Y_{t-1} \\
I P C_{t-1} \\
M 1_{t-1}
\end{array}\right)+\left(\begin{array}{c}
U_{1 t} \\
U_{2 t} \\
U_{3 t} \\
U_{4 t} \\
U_{5 t} \\
U_{6 t}
\end{array}\right)
$$

Adicional, se plantea la realización de test de raíz unitaria, diagnóstico, errores y estabilidad del modelo, cuya descripción metodológica puede ser consultado en el anexo ${ }^{2}$ estadístico digital, en los apartados 1.1 y 1.2 .

\section{TEST DE CAUSALIDAD DE GRANGER}

Esta prueba sirve para determinar si dadas un par de variables aleatorias, es posible verificar que variable antecede a la otra, si el comportamiento actual o pasado de una variable $x$ causa en el sentido de Granger el comportamiento de una variable $y$, además de verificar si este efecto tiene una causalidad unidireccional $(x \rightarrow y)$ o también puede ser bidireccional ( $x$ $\leftrightarrow y$ ) (Granger, 1969).

Según Gujarati y Porter (2010), la prueba de causalidad unidireccional $(x \rightarrow y)$ en el sentido de Granger implica una estimación de la siguiente regresión:

$$
X_{t}=\sum_{l=1}^{n} \alpha_{l} Y_{t-\imath}+\sum_{l=1}^{n} \beta_{l} X_{t-\jmath}+u_{1 t}
$$

Y para una la contrastación de una causalidad bidireccional $(x \leftrightarrow \rightarrow y)$ en el sentido de Granger, implica la estimación de las siguientes regresiones:

$$
\begin{aligned}
& X_{t}=\sum_{\imath=1}^{n} \alpha_{\imath} Y_{t-\imath}+\sum_{\imath=1}^{n} \beta_{l} X_{t-\jmath}+u_{1 t} \\
& Y_{t}=\sum_{l=1}^{n} \gamma_{\iota} Y_{t-\imath}+\sum_{l=1}^{n} \delta_{l} X_{t-\jmath}+u_{2 t}
\end{aligned}
$$

El planteamiento de las pruebas específicas utilizando las variables descritas para el presente estudio, puede ser consultado en la tabla 1 del anexo estadístico digital, en el apartado 1.3.

\section{FUNCIÓN IMPULSO RESPUESTA}

La ventaja de utilizar el modelo var es que éste introduce particularidades como la causalidad y la exogeneidad, esto permite capturar el comportamiento de las variables ante un choque externo a través del tiempo, esto se conoce como la Función Impulso Respuesta (FIR), lo cual 
Tabla 2: Test de Granger

\begin{tabular}{|c|c|c|c|}
\hline Ecuación & Excluido & Prob $>$ chi 2 & Conclusión \\
\hline $\mathrm{BM}$ & $\mathrm{CB}$ & 0.016 & $\mathrm{CB} \square \mathrm{BM}$ \\
\hline $\mathrm{BM}$ & IPC & 0.018 & $\mathrm{IPC} \square \mathrm{BM}$ \\
\hline $\mathrm{CB}$ & $\mathrm{Y}$ & 0.024 & $\mathrm{Y} \square \mathrm{CB}$ \\
\hline $\mathrm{CB}$ & IPC & 0.012 & $\mathrm{IPC} \square \mathrm{CB}$ \\
\hline M1 & $\mathrm{BM}$ & 0.011 & $\mathrm{BM} \square \mathrm{M} 1$ \\
\hline M1 & $\mathrm{Y}$ & 0.004 & $\mathrm{Y} \Rightarrow \mathrm{Ml}$ \\
\hline
\end{tabular}

Nota: resultados de la prueba de causalidad de Granger obtenidos a través del software Stata 14 (solo se incluyen los resultados de causalidad positivos).

logra que se capture la dinámica de las interrelaciones y el movimiento simultáneo de las variables (Gutiérrez y Rocío, 2012). Es decir, la función de impulso-respuesta puede capturar la reacción de cada una de las variables ante un shock proveniente de innovaciones estructurales, para cada una de estas innovaciones dentro del modelo hay tantas funciones de impulsorespuesta como variables endógenas, para analizar de mejor manera esta reacción de las variables ante shocks externos se lo puede realizar a través de un gráfico, en los que se observa dicha respuesta a través del tiempo (Novales, 2017).

\section{RESULTADOS Y ANÁLISIS}

Los resultados del test de raíz unitaria, estacionalidad, criterio de selección de rezagos, pruebas de diagnóstico, errores, estabilidad del modelo y la estimación del modelo var se puede consultar en el anexo estadístico digital, en el apartado del 2.1 hasta el 2.5 .

\section{RESULTADOS DE PRUEBA DE CAUSALIDAD DE GRANGER}

Se procede a aplicar la prueba de causalidad de Granger que permitirá determinar las hipótesis monetarias planteadas - monetarista, horizontalista, estructuralista y de preferencia de liquidez- y detalladas en la tabla 1, que pueden ser constatadas con la evidencia empírica recolectada, cuyos principales resultados se detallan en la tabla (ver Tabla 2). Adicional, los resultados específicos de las pruebas de causalidad de Granger pueden ser revisados en la tabla 10 del anexo estadístico digital, del apartado 2.6 .

El primer resultado relevante obtenido es la causalidad unidireccional entre $\mathrm{CB} \rightarrow \mathrm{BM}$, lo que confirma la primera causalidad de la perspectiva horizontalista; en segundo lugar, se obtiene una causalidad unidireccional de $\mathrm{Y} \rightarrow \mathrm{M} 1$ que iría acorde con el mismo enfoque y, por último, se obtiene una causalidad entre $\mathrm{CB} \rightarrow \mathrm{M} 1$, sin embargo, esta causalidad no es directa, sino más bien se logra a través de la $\mathrm{BM}$ de la siguiente manera: $\mathrm{CB} \rightarrow \mathrm{BM} \rightarrow \mathrm{M}$. Las pruebas de causalidad de Granger, si bien están enfocadas en identificar causalidades directas, éstas no permiten rechazar la existencia de causalidades indirectas (Balacco, 1986), por lo cual, la existencia de esta causalidad indirecta podría brindar una aproximación para confirmar la segunda hipótesis del mismo enfoque. 
Al cumplirse las tres hipótesis de la vertiente horizontalista de endogeneidad monetaria, se confirma la prevalencia de esta dinámica para la economía ecuatoriana, esto permite brindar una aproximación teórica sobre el rol del Banco Central, el posible uso de la tasa de interés y si se toma en cuenta o no la preferencia por la liquidez de los agentes. Según la vertiente horizontalista la banca central cubre totalmente las reservas de liquidez de la banca comercial y es su prestamista de última instancia, así mismo, utiliza la tasa de interés como herramienta de corto plazo para controlar posibles problemas de liquidez, es así como crea una relación elástica entre la tasa de interés y la demanda de crédito bancario, además de no tomar en cuenta la preferencia de liquidez de los agentes en el proceso de creación monetaria endógena.

Adicional, se encuentra una causalidad entre el IPC $\rightarrow$ BM, IPC $\rightarrow$ Св Y Ү $\rightarrow$ CB, dichas causalidades podrían no encajan con los enfoques monetarios planteados, pero muestran una relevancia empírica cuyo análisis puede ser ampliado en otros estudios.

\section{RESULTADO DE FUNCIÓN IMPULSO RESPUESTA}

Una vez obtenidos los resultados de las pruebas de causalidad de Granger, se procederá a presentar los resultados de la función impulso-respuesta de aquellas variables relevantes para esta sección del estudio, éstas son: crédito bancario (Св) sobre oferta monetaria (M1) y PIB real (Y) sobre oferta monetaria (M1). Como se observa en la figura (ver Figura 1), frente a un shock en el crédito bancario tiene una respuesta inicial positiva sobre la oferta monetaria, este efecto dura aproximadamente un trimestre y para el cuarto trimestre se estabiliza. Así mismo, se observa la respuesta de la oferta monetaria frente a un shock del PIB real, según la figura (ver Figura 2) la oferta monetaria tiene una respuesta positiva cuyo pico llega aproximadamente en el primer trimestre y llega a estabilizarse para el tercer trimestre.

Estos resultados concuerdan con lo obtenido en las pruebas de causalidad de Granger, pues evidencian que ante un shock tanto en el св у у, se obtiene una respuesta positiva en $\mathrm{M1}$, aunque se destaca que se observa un impulso más fuerte proveniente de un shock en $\mathrm{Y}$.

\section{DISCUSIÓN}

Los resultados hallados en la presente investigación confirman lo hallado en el estudio de Pérez y Guncay (2019), la hipótesis de endogeneidad monetaria está presente en ambos estudios a pesar de haber utilizado variables distintas para las pruebas de causalidad de Granger y de haber utilizado un modelo con ciertas diferencias como se detalló anteriormente.

El presente estudio no abarca la relación entre el proceso de oferta monetaria y el saldo de la balanza de pagos, sin embargo, el estudio antes mencionado si lo abarca, en él se halla que el proceso de oferta monetaria no está condicionado al resultado de balanza de pagos, esto no quiere decir que no tenga importancia, sino más bien que el crédito que se otorgue como resultado de la actividad económica tiene un mayor efecto sobre la oferta monetaria que el resultado de la balanza de pagos (Pérez y Guncay, 2019). Así mismo, el presente estudio evaluó las funciones impulso-respuesta tanto del crédito bancario sobre la oferta monetaria como del PIB real sobre la oferta monetaria, los resultados obtenidos en el presente trabajo difieren con los obtenidos en el anterior mencionado, pues éste detalla la respuesta positiva de un shock ante el 
Figura 1: FIR del crédito bancario sobre la oferta monetaria

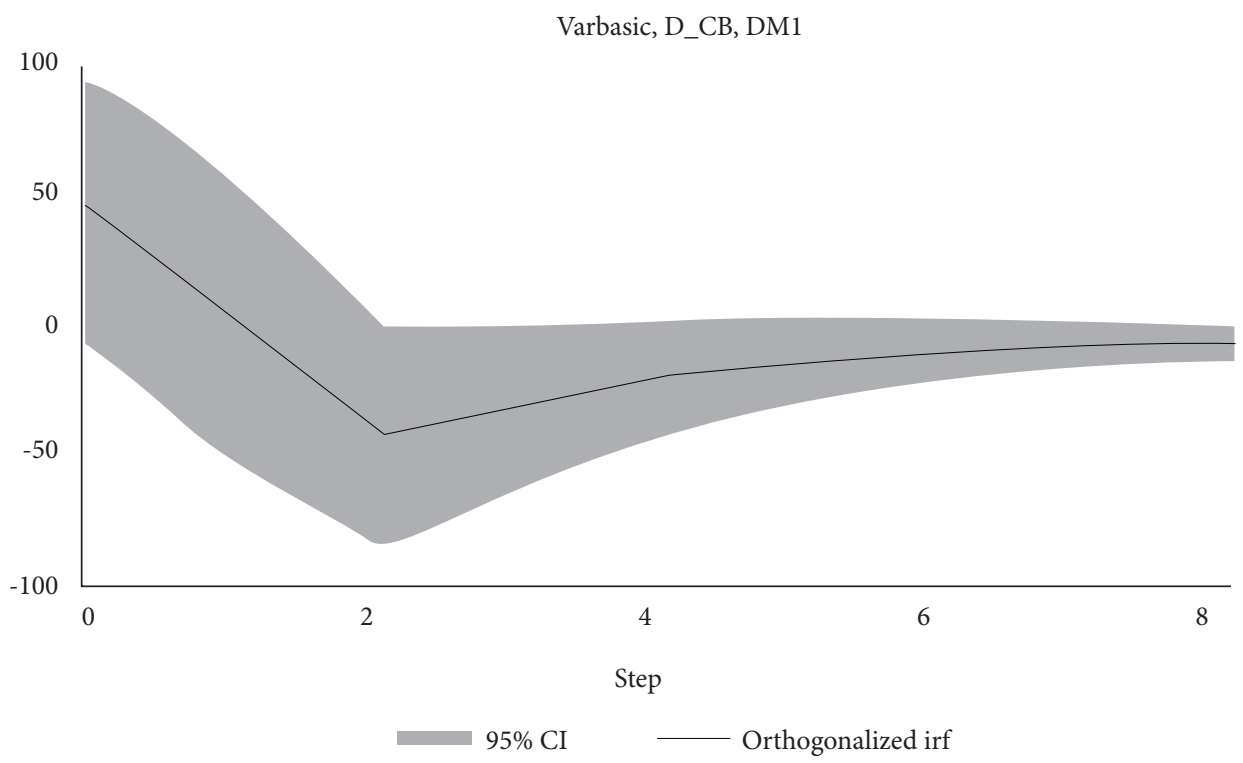

Fuente: Stata 14 .

Figura 2: FIR del PIB real sobre la oferta monetaria

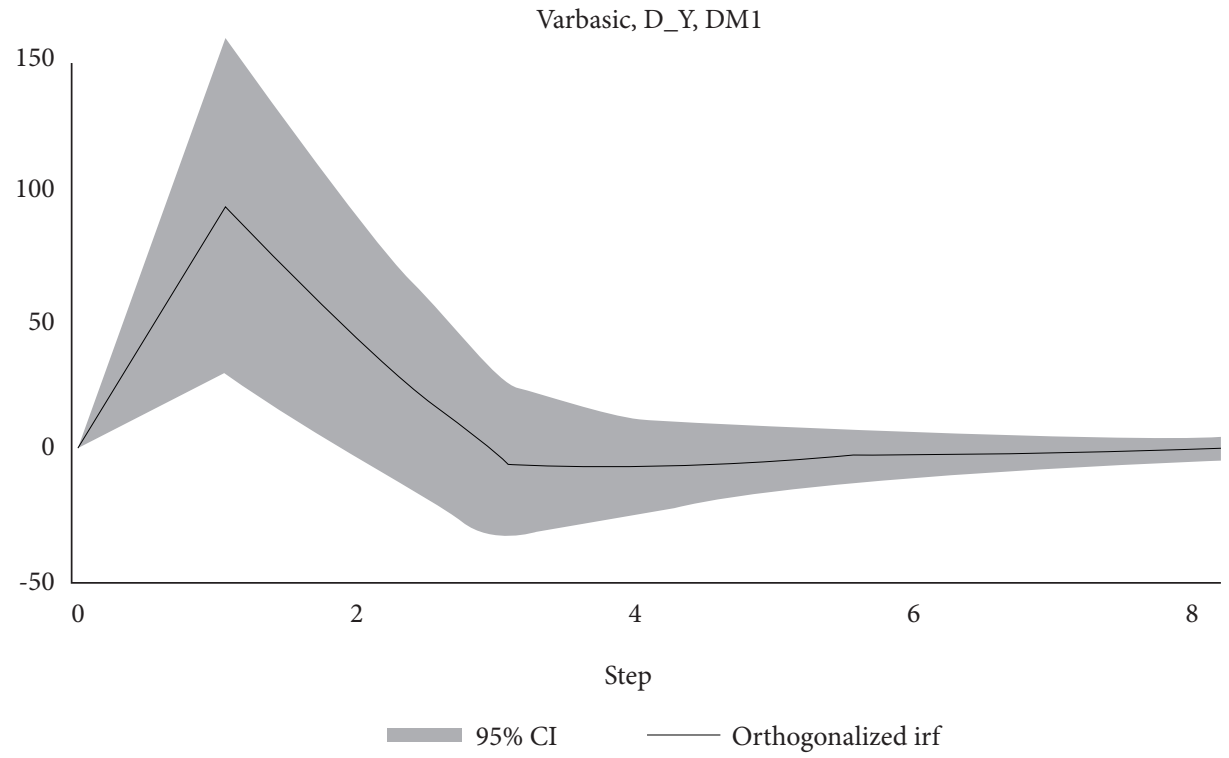

Fuente: Stata 14 . 
crédito bancario sobre la oferta monetaria que dura aproximadamente cuatro trimestres, mientras que el estudio antes mencionado el efecto se prolonga por alrededor de cinco trimestres.

Uno de los limitantes del presente estudio y de los estudios encontrados hasta la actualidad, es que no se contempla la posibilidad de un proceso de fuga de capitales producto de un comportamiento totalmente acomodatista de la banca central, esta relación y sus implicaciones de política económica se podrían profundizar en investigaciones posteriores.

Los resultados obtenidos en la presente investigación que confirman la presencia de la dinámica horizontalista (acomodatista), presentan ciertas complicaciones en una economía dolarizada. En primer lugar, bajo el régimen monetario de dolarización se elimina la posibilidad de que el Banco Central sea prestamista de última instancia para la banca comercial, más bien, desde la imposición de la dolarización en el año 2000, el BCE ha optado por la alimentación de fondos de liquidez externos, coeficientes de liquidez doméstica y encaje bancario, que sustituyan así la figura del prestamista de última instancia (Carrasco, 2015), pero de igual manera en la Ley de Fomento Productivo (2018) se eliminó la posibilidad de mantener el coeficiente de liquidez doméstica cortando definitivamente esta capacidad, mostrando así un contraste entre el comportamiento actual y la teoría existente.

En segundo lugar, si bien legalmente el все tiene la capacidad de manejar la tasa de interés, esto solo llega a ser una formalidad, ya que en la práctica tan solo llega a fijar la tasa de interés referencial con información proporcionada por la banca comercial, es decir, es la banca comercial quien fija las tasas de interés y el всE establece un techo de esta tasa, tomando en cuenta a la tasa de interés activa vigente en la última semana completa del mes anterior con un recargo del 50\% (Carrasco, 2015). Esto muestra la segunda disparidad entre el comportamiento teórico encontrado y la práctica real.

\section{CONCLUSIONES}

La presente investigación ratificó la hipótesis monetaria de endogeneidad del dinero, lo cual indica que a pesar de que la economía ecuatoriana con su particularidad de economía dolarizada, ésta va acorde con los postulados poskeynesianos, que indican que el proceso de creación monetaria ocurre por las necesidades de producción o consumo de los hogares y empresas, el mismo que es canalizado a través del crédito bancario.

Dentro de la visión de endogeneidad monetaria, las pruebas de causalidad en el sentido de Granger confirmaron la postura de la vertiente horizontalista (acomodatista), esto en base a la evidencia empírica encontrada, que detalla la existencia de una causalidad unidireccional desde el crédito bancario a la base monetaria, la presencia de una causalidad indirecta desde el crédito bancario hacia la oferta monetaria y por último se encontró una causalidad unidireccional desde el PIB real a la oferta monetaria.

La confirmación teórica de la vertiente horizontalista sobre la dinámica monetaria de la economía ecuatoriana, podría dar una idea del funcionamiento del proceso de la oferta monetaria en el país y el rol que toma el Banco Central dentro de este proceso. Teóricamente, la banca central cubre totalmente las necesidades de reservas de la banca comercial y funge el papel de prestamista de última instancia de éstas, además, podría utilizar la tasa de interés de 
corto plazo para controlar posibles problemas de liquidez, creando así una relación completamente elástica entre la tasa de interés y la demanda de crédito bancario, por último, no se toma en cuenta la preferencia por la liquidez de los agentes en el proceso de oferta monetaria vía crédito bancario, pues la banca comercial mantiene un comportamiento pasivo ante la demanda del crédito.

La función de impulso respuesta aquí detallada, obtienen una respuesta positiva tanto del crédito bancario y el PIB real sobre la oferta monetaria, el shock sobre el crédito bancario dura aproximadamente cuatro trimestres hasta que la serie vuelve a su estado estacionario; mientras que el shock sobre el PIB real se prolonga por aproximadamente tres trimestres. Se debe destacar que la respuesta de la oferta monetaria sobre el shock del pIB real es mayor que sobre el crédito bancario.

Pese a que se realizó una aproximación teórica con base en la evidencia empírica encontrada, el régimen dolarizado ecuatoriano muestra ciertas diferencias entre la descripción teórica y el desenvolvimiento del esquema monetario vigente, tanto en la parte de normativa legal que impide la existencia de un prestamista de última instancia o de un instrumento que se aproxime y de la no utilización de la tasa de interés por parte del BCE para la regulación discrecional del crédito bancario, a pesar que sí está facultado para hacerlo.

La confirmación de un proceso de oferta monetaria endógena en una economía dolarizada que es canalizada a través del crédito bancario, debe ser manejado con prudencia por parte de las autoridades monetarias del país, ya que todavía no se conoce a profundidad las implicaciones que esto conlleva y hasta qué punto puede ser manejado el proceso de oferta monetaria, lo cual obliga a realizar investigaciones que profundicen esta problemática a fin de tener una visión más clara para el manejo de la política económica.

\section{NOTAS}

1 Se agradece a Bryan Pérez, Eric Meyer y a dos evaluadores anónimos quienes, con sus sugerencias y comentarios a versiones preliminares de este artículo, ayudaron a mejorarlo notablemente.

2 Para acceder al Anexo digital de este artículo, diríjase al siguiente enlace: https://uceedu-my.sharepoint.com/:b:/g/personal/revistaeconomia_uce_edu_ec/ed6ghfwrsonpg_doF5qv8liB8wocmB17cktsL6 Rcf6znrg?e=a7ylct

\section{REFERENCIAS}

Acosta, A. (2001). La triste aventura de la dolarización. Iberoamericana, 1(3), 180-188.

Alvarado, F. (2011). La hipótesis postkeynesiana del dinero endógeno: evidencia empírica para Colombia 1982-2009. Ensayos de Economía, 21(38), 45-83.

Asamblea Constituyente de la República del Ecuador (2008). Constitución de la República del Ecuador. Quito, Ecuador: Registro Oficial.

Asamblea Nacional del Ecuador. (2016). Código Orgánico Monetario y Financiero. (s. d.).

Asamblea Nacional del Ecuador. (2018). Ley para Fomento Productivo, Atracción de inversiones y Generación de empleo. Quito, Ecuador: Registro Oficial.

Bain, K. and Howells, P. (2003). Monetary economics: policy and its theoretical basis. London, UK: Palgrave Macmillan. 
Balacco, H. R. (1986). Algunas consideraciones sobre la definición de causalidad de Granger en el análisis econométrico. Recuperado de https://aaep.org.ar/anales/works/works1986/balacco.pdf

Brue, S. y Grant, R. (2009). Historia del Pensamiento Económico. México, México DF: Cengage Learning.

Carrasco, A. (2015). La política monetaria en el Ecuador con régimen cambiario dolarizado. Revista Fiscalidad, 10, 8-53.

Chick, V. and Dow, S. (2002). Monetary policy with endogenous money and liquidity preference: a nondualistic treatment. Journal of PostKeynesian Economics, 24(4), 587-607.

Davidson, P. (2002). Dolarización, las funciones de un Banco Central y la economía ecuatoriana. Cuestiones Económicas, 18(3), 55-77.

Friedman, M. (1977). Nobel Lecture: Inflation and Unemployment. Journal of Political Economy, 85(3), 451-472. Recuperado de https://doi.org/10.1086/260579

Garzón, I. D. V. (2009). Oferta monetaria y tasa de interés: un análisis comparativo en las teorías postkeynesiana y circuitista. Ecos de Economía: A Latin American Journal of Applied Economics, 13(29), 21-59.

Granger, C. W. J. (1969). Investigating causal relations by econometric models and cross-spectral methods. Econometrica, 37(3), 424-438. Recuperado de https://doi.org/10.2307/1912791

Gujarati, D. y Porter, D. (2010). Econometría. México, México DF: McGraw-Hill/Interamericana Editores, S. A.

Gutiérrez, P. y Rocío, A. (2012). Pronóstico de la inflación ecuatoriana mediante vectores autorregresivos estructurales. Recuperado de http://bibdigital.epn.edu.ec/handle/150oo/4496

Haghighat, J. (2011). Endogenous and exogenous money: an empirical investigation from Iran. Journal of Accounting, Finance and Economics, 1(1), 61-76.

Keynes, J. M. (1933). Una teoría monetaria de la producción. Cuadernos de economía, 17(28), 246-249.

Keynes, J. M. (1936). Teoría general de la ocupación, el interés y el dinero. México, México DF: Fondo de Cultura Económica.

Lavoie, M. (1992). Foundations of Post-Keynesian Economic Analysis. Recuperado de https://ideas.repec. org/b/elg/eebook/275.html

Mántey, G. (1997). Lecciones de economía monetaria. México, México DF: Universidad Nacional Autónoma de México.

Moore, B. J. (1991). Money Supply Endogeneity: «Reserve Price Setting» or «Reserve Quantity Setting»? Journal of Post Keynesian Economics, 13(3), 404-413.

Naranjo, M. (2003). La dolarización de la economía del Ecuador: Tres años después. Cuestiones Económicas, 19(1). Recuperado de https://www.bce.fin.ec/cuestiones_economicas/images/ PDFS/2003/No1/Vol.19-1-2003MARCONARANJO.pdf

Novales, A. (2017). Modelos vectoriales autoregresivos (VAR). Recuperado de https://www.ucm.es/data/ cont/media/www/pag-41459/ var.pdf

Palley, T. I. (1996). Accommodationism versus structuralism: time for an accommodation. Journal of Post Keynesian Economics, 18(4), 585-594.

Palley, T. I. (1998). Accommodationism, structuralism, and superstructuralism. Journal of Post Keynesian Economics, 21(1), 171-173.

Pastrana, W. O. (2018). La eficacia de la política monetaria, cuando el dinero es endógeno. Un enfoque postkeynesiano para la economía boliviana. (s. d.). 
Paula, L. F. R. de. (2003). Teoria horizontalista da moeda e do crédito: Crítica da crítica. Estudos Econômicos, 33(2), 287-323. Recuperado de https://doi.org/10.1590/So101-41612003000200004

Pérez, D. y Guncay, C. (2019). Endogeneidad de la oferta monetaria en Ecuador: un análisis desde la visión post-keynesiana. Cuestiones Económicas, 29(1), 73-114.

Piégay, P. y Rochon, L.-P. (2006). Teorías monetarias postkeynesianas. Madrid, España: Ediciones AKAL.

Rachma, M. S. (2010). Endogeneity of Indonesian money supply. Economic Journal of Emerging Markets, 2(3), 277-288. Recuperado de https://doi.org/10.20885/ejem.v2i3.2313

Rodríguez, C., Padrón, D. y Olivera, A. (2002). La endogeneidad de la oferta monetaria: teoría y evidencia empírica para la economía española. RAE: Revista Asturiana de Economía, (29), 91-110.

Shanmugam, B., Nair, M. and Ong, W. L. (2003). The endogenous money hypothesis: Empirical evidence from Malaysia (1985-2000). Journal of Post Keynesian Economics, 25(4), 599-611. Recuperado de https://doi.org/10.1080/01603477.2003.11051378

Vega Miranda, F. y Lechuga, J. (2018). Dinero endógeno y exógeno. Revista de Economía, 37, 30-65.

Villalba, M. (2019). Dos décadas de dolarización ¿qué hemos aprendido sobre este esquema monetario? En Economía y Territorio, Dolarización: dos décadas después (pp. 7-42). Quito, Ecuador: Abya Yala.

Wray, L. R. (2007). A Post-Keynesian view of Central Bank independence, policy targets, and the rules-versus-discretion debate. Working Paper n. ${ }^{\circ}$ 510. Recuperado de https://doi.org/10.2139/ssrn.1006092 
